\title{
Should screening of genital infections be part of antenatal care in areas of high HIV prevalence? A prospective cohort study from Kigali, Rwanda, 1992-1993
}

Valeriane Leroy, Andre De Clercq, Joel Ladner, Jos Bogaerts, Philippe Van de Perre, François Dabis, for the Pregnancy and HIV (EGE) Group
sub-Saharan Africa. ${ }^{1}$ In Rwanda, a national serosurvey estimated in December 1986 that the HIV seroprevalence was $18 \%$ among urban dwellers, reaching $30 \%$ among those aged $26-40$ years and $21 \%$ specifically among adult women, all ages combined. ${ }^{2}$ Heterosexual transmission accounts for most of the cases of HIV infection in sub-Saharan Africa and there are more women of reproductive age and children infected on this continent than in any other part of the world. ${ }^{13}$ HIV infection has therefore common epidemiological features with other sexually transmitted diseases (STDs). ${ }^{4}$ Because STDs are a major cause of chorioamnionitis which is itself a risk factor for mother-to-child transmission of HIV infection, ${ }^{5}$ their association during pregnancy could modify the rate of motherto-child transmission of HIV. A prospective cohort study on the impact of HIV-1 infection on pregnancy was launched in July 1992, in Kigali, the capital city of Rwanda. ${ }^{6}$ The main objectives were: 1) to document the pregnancy outcomes after screening for common genital infections and treating them and 2) to study the maternal post-partum complications in HIV-positive (HIV + ) women in comparison to HIV-negative (HIV-) women.

The aim of the present report is to provide background prevalence figures of maternal genital infections, to estimate the incidence of genital infections until delivery and to study the relation between HIV and other genital infections, in this prospective cohort study, in order ultimately to improve STDs and HIV prenatal control programmes in this African environment. Neisseria gonorrhoea $(1 \cdot 0-13 \cdot 3), 2 \cdot 6$ for Trichomonas vaginalis $(1 \cdot 5-4 \cdot 6)$ and 1.6 for Candida vaginalis $(1 \cdot 1-2 \cdot 4)$.

Conclusion-In the context of high HIV-1 seroprevalence among pregnant women, prenatal care should include at least once screening for genital infections by clinical examination with speculum and $a$ syphilis testing in Africa.

(Genitourin Med 1995;71:207-211)

Keywords: STDs; genital infections; HIV-1 infection; Africa; pregnant women

\section{Introduction}

Human immunodeficiency virus type-1 (HIV) infection is a major public health problem in

\section{Subjects and methods}

A prospective cohort has been enrolled at the July 1992 and August 1993. ${ }^{7}$ All pregnant women attending the prenatal clinic of the $\mathrm{CHK}$, living in the Kigali area and wishing to deliver at the CHK were informed by a trained social worker about HIV infection as well as objectives, constraints and benefits of the study. Eligible women were examined by echography. Those between 21 and 28 weeks of gestation and consenting to participate to the study were systematically offered HIV antibody screening by two commercial enzyme-linked immuno-sorbent assays (ELISA) (Vironostika HIV Mixt and Centre Hospitalier de Kigali (CHK) between 
Vironostika Uniform, Organon Teknika, Boxtel, the Netherlands). Discordant samples by ELISA were confirmed by a commercial Western Blot technique (Du Pont de Nemours, Delaware, USA) using the Centers for Disease Control criteria of interpretation. ${ }^{8}$ Pre and post-test counselling were systematically offered.

Two weeks after the HIV screening test, we enrolled formally. all HIV+ women and an equivalent number of HIV - women. The two groups were selected to be comparable in terms of maternal age and parity. At inclusion, data on the socio-economic status and on gynaecological and medical history were collected on standardised questionnaires. History of treated leucorrhoea and genital ulcers during the two years preceding inclusion was recorded. A clinical examination was performed by a physician, looking for HIVrelated signs and symptoms and particularly those included in the WHO clinical AIDS case definition. ${ }^{9}$ A pelvic examination was also performed. Physicians, nurses and social workers were blinded to the HIV serostatus. CD4 and CD8 lymphocyte absolute counts were performed using a commercial immunomagnetic method (Biosys, France). A Rapid Plasma Reagin (RPR, Syphacard, Wellcome, USA) serum test was used for the diagnosis of syphilis. ${ }^{10}$ It was considered positive when serum was reactive at dilution 1:2. ${ }^{11}$ Chlamydiae trachomatis was detected on cervical smear by an enzyme immunoassay (EIA Chlamydiazyme, Syva, USA). Gonococcal laboratory culture as well as direct microscopic examination by saline wet mount for trichomonas and candida (yeast) were also performed. Standardised treatment was systematically offered in case of genital infection to women and their partners. (Treatment modalities: in case of syphilis: benzathinepenicillin, 2-4 million units, one injection each week during three weeks; Neisseria gonorrhoea: spectinomycine, $2 \mathrm{~g}$ in one injection; Chlamydiae trachomatis and chancroid ulcers: erythromycin, $1.5 \mathrm{~g}$ daily per os during 7 days; trichomoniasis: metronidazole, $2 \mathrm{~g}$ single dose per os (only during second and third trimester); candidiasis: myconazole, one vaginal suppository of $400 \mathrm{mg}$ daily during three days.) Until delivery, each woman enrolled had a monthly follow-up including a systematic pelvic examination. Screening of genital infections was repeated, except for chlamydiae, in the following situations: (1) if control was required at the end of the treatment, (2) if symptoms or abnormal physical signs at pelvic examination were found (leucorrhea, genital ulcer, cervical inflammation), or (3) if women complained of genital symptoms. Treatment procedures were repeated if needed.

HIV+ and HIV - women were compared at inclusion for socio-economic and clinical characteristics and prevalence of genital infections using the chi square, Fisher's and Student's $t$ tests with a significance level of $5 \%$. In each group of women, we estimated the incidence density rate (incidence) of individual genital infections until delivery except for Chlamydia trachomatis. Women who presented with syphilis at inclusion were excluded from this longitudinal analysis because of the persistence of positive markers during six months or more after treatment.

Relative Risks (RR) and prevalence odds ratios (OR) were computed with their 95\% confidence interval (CI). Adjustment was performed with the Mantel-Haenszel method and their 95\% CI computed with the Miettinen method.

\section{Results}

From 1 July 1992 to 13 August 1993, 1233 pregnant women attending the antenatal clinic of the CHK and fulfilling the inclusion criteria were tested for HIV-1 antibodies. Among them, $424(34.4 \%)$ were found to be HIV+ (CI: $31 \cdot 7 \%-37 \cdot 1 \%$ ). Forty HIV+ women $(9.4 \%)$ could not be enrolled in the cohort, mainly because of husband refusal or change of residence. When looking, for comparable HIV - women, 25 could not be enrolled for the same reasons.

In total, 15 days after the first interview, $384 \mathrm{HIV}+$ women and $381 \mathrm{HIV}$ - women were enrolled in the cohort $(92.2 \%$ of eligible women). The mean maternal age was 26.0 years (standard deviation (SD): $4 \cdot 8$, range: 15-43). The average number of pregnancies, including the current one, was 2.5 (SD: 1.7 , range: 1-11). The proportion of women living in a stable relationship (legal marriage or common law union) was not different between the HIV+ and HIV - groups (93.4\% versus $91.2 \%$, chi square test; $\mathrm{p}=0.23$ ). HIV+ women did not differ from HIVwomen in terms of occupation, $56.9 \%$ being housewives overall. The HIV+ women enrolled had a lower educational level than HIV - women: $42.4 \%$ of the HIV+ women had a primary school level only versus $29.3 \%$ (chi square test; $p=0.0001$ ). Socioeconomic characteristics will be described in detail elsewhere.

When comparing clinical signs and symptoms of the AIDS clinical definition at inclusion, the only differences between HIV+ and HIV - women were for generalised lymphadenopathy $(10 \cdot 7 \%$ versus $1 \cdot 1 \%$ respectively; chi square test; $p=0.0001$ ) and recurrent herpes zoster infection $(3.6 \%$ versus $0.3 \%$; Fisher's exact test; $p=0.0007)$. At enrolment in the cohort, three HIV+ women only fulfilled the WHO definition for clinical AIDS and none of the HIV - women. Profound immunosuppression defined by a CD4 count lower than $200 / \mathrm{mm}^{3}$ was observed in $7.5 \%$ of the HIV+ women $(n=360)$ and in $0.3 \%$ of the HIV - women $(n=362)$, (chi square test; $\mathrm{p}<0.0001$ ).

A history of leucorrhoea and genital ulcers was more frequently reported by HIV + women than by HIV - women (table 1). The same difference was observed with clinical signs at pelvic examination (table 1). Among the genital infections screened, candidiasis excluded and both groups combined, $25 \cdot 2 \%$ of the women presented with at least one 
Table 1 Gynaecological history and clinical signs among $H I V-1$ infected (HIV+) and uninfected (HIV-) prgenant women at inclusion in the cohort (results are percentages). Kigali, Rwanda, 1992-1993

\begin{tabular}{|c|c|c|c|}
\hline & $\begin{array}{l}H I V+ \\
(n=384)\end{array}$ & $\begin{array}{l}H I V- \\
(n=381)\end{array}$ & $p$ value \\
\hline $\begin{array}{l}\text { History of treated leucorrhoea } \\
\text { History of genital ulcers } \\
\text { Leucorrhoea at pelvic examination } \\
\text { Genital ulcers } \\
\text { Cervical inflammation } \\
\text { Cervical contact bleeding }\end{array}$ & $\begin{array}{r}14 \cdot 3 \\
22 \cdot 3 \\
48 \cdot 2 \\
7 \cdot 6 \\
34 \cdot 4 \\
49 \cdot 5\end{array}$ & $\begin{array}{r}5 \cdot 3 \\
8 \cdot 4 \\
36 \cdot 1 \\
3 \cdot 7 \\
15 \cdot 7 \\
29 \cdot 2\end{array}$ & $\begin{array}{l}0 \cdot 0001 \\
0 \cdot 0001 \\
0 \cdot 0007 \\
0 \cdot 032 \\
0 \cdot 0001 \\
0 \cdot 0001\end{array}$ \\
\hline
\end{tabular}

^In the two years preceding inclusion.

tChi square test.

Table 2 Prevalence of genital infections among $H I V-1$ infected $(H I V+)$ and uninfected (HIV-) pregnant women at inclusion in the cohort (results are percentages). Kigali, Rwanda, 1992-1993

\begin{tabular}{lccl}
\hline Genital infections & $\begin{array}{c}H I V+ \\
(n=384)\end{array}$ & $\begin{array}{c}H I V- \\
(n=381)\end{array}$ & $p$ value* \\
\hline Syphilis & $6 \cdot 3$ & $3 \cdot 7$ & $0 \cdot 13$ \\
Neisseria gonorrhoea & $7 \cdot 0$ & $2 \cdot 4$ & $0 \cdot 0056$ \\
Trichomonas vaginalis & $20 \cdot 2$ & $10 \cdot 9$ & $0 \cdot 0007$ \\
Chlamydia trachomatis & $3 \cdot 4$ & $5 \cdot 5$ & $0 \cdot 21$ \\
Candida albicans & $22 \cdot 3$ & $20 \cdot 1$ & $0 \cdot 49$ \\
At least one of the above genital & $30 \cdot 2$ & $20 \cdot 2$ & $0 \cdot 0019$ \\
infections, candidiasis excepted & & & \\
\hline
\end{tabular}

^Chi square test. physical sign or symptom at pelvic examination (leucorrhoea or genital ulcer or cervical inflammation) (table 3). Among the 67 women who had syphilis or Neisseria gonorrhoea infection, $70 \%$ presented at least one abnormal physical sign or symptom. Thus, the positive predictive value of the clinical examination was $88 \%$ and the negative predictive value was $94 \%$ for these two major STDs.

At inclusion, regardless of HIV status, there was no significant difference in the average number of $\mathrm{CD} 4$ lymphocytes among women presenting at least one genital infection and the others (628 and $670 \mathrm{CD} 4$ cells $/ \mathrm{mm}^{3}$, respectively, Student's $t$ test, $\mathrm{p}=0 \cdot 12$ ). When adjusting for HIV serostatus, there was no significant association between immunodeficiency (CD4 cell count lower than $200 / \mathrm{mm}^{3}$ ) and the presence of genital infections (adjusted OR: 1·14; CI: 0.53-2.45).

Over the study period, the mean follow-up of the cohort was 70 days until delivery (SD: 8 days; range: 0-128) without difference between the two groups. There was no significant difference between HIV+ and HIVwomen for the incidence of active syphilis, excluding those who were diagnosed at inclusion (table 4). There also was no significant difference between the two groups for the incidence of gonorrhoea (table 4). There was a significantly higher risk of trichomoniasis and candidiasis among HIV+ women than HIV - women (table 4).

In our study, only one of the $26 \mathrm{HIV+}$ women versus one of the nine HIV - women had repeated gonorrhoea infection after a standardised treatment (Fisher's exact test, p $=0.41$ ); among those who presented with a vaginal trichomoniasis infection at inclusion, $22.4 \%$ of the $76 \mathrm{HIV}+$ women were found positive during follow-up versus $7 \cdot 3 \%$ of the $41 \mathrm{HIV}$ - women (chi square test, $\mathrm{p}=0.03$ ); finally, $22.6 \%$ of the $84 \mathrm{HIV}+$ women who had a vaginal candidiasis at inclusion had a new episode versus $25.3 \%$ of the $75 \mathrm{HIV}-$ women (chi square test, $\mathrm{p}=0 \cdot 68$ ).

Table 3 Prevalence of clinical signs * among the 316 pregnant women presenting at least one genital infection at inclusion in the cohort. Kigali, Rwanda, 1992-1993

\begin{tabular}{llllll}
\hline Genital infections & $\begin{array}{l}\text { Number of } \\
\text { events }\end{array}$ & $\begin{array}{l}\text { Leucorrhoea } \\
(\%)\end{array}$ & $\begin{array}{l}\text { Genital ulcers } \\
(\%)\end{array}$ & $\begin{array}{l}\text { Cervical inflammation } \\
(\%)\end{array}$ & $\begin{array}{l}\text { At least one } \\
\text { clinical sign (\%) }\end{array}$ \\
\hline Syphilis & $38(100 \%)$ & $60 \cdot 5$ & $13 \cdot 1$ & $28 \cdot 9$ & $71 \cdot 1$ \\
Neisseria gonorrhoea & $35(100 \%)$ & $60 \cdot 0$ & $14 \cdot 3$ & $35 \cdot 2$ & $68 \cdot 6$ \\
Trichomonas vaginalis & $117(100 \%)$ & $76 \cdot 1$ & $9 \cdot 4$ & $38 \cdot 4$ & $82 \cdot 1$ \\
Chlamydia trachomatis & $34(100 \%)$ & $50 \cdot 0$ & $5 \cdot 8$ & $20 \cdot 6$ & $58 \cdot 8$ \\
Candida albicans & $159(100 \%)$ & $72 \cdot 6$ & $6 \cdot 4$ & $28 \cdot 5$ & $76 \cdot 7$ \\
\hline
\end{tabular}

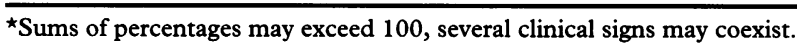

Table 4 Incidences of genital infections per 100 women-months among HIV-1 infected (HIV+) and uninfected (HIV-) pregnant women during the last three months of pregnancy. Kigali, Rwanda, 1992-1993

\begin{tabular}{|c|c|c|c|c|}
\hline Genital infections & $\begin{array}{l}H I V+\star \\
(n=360)\end{array}$ & $\begin{array}{l}H I V-\star \\
(n=367)\end{array}$ & $\begin{array}{l}\text { Relative } \\
\text { risk }\end{array}$ & $\begin{array}{l}95 \% \text { Confidence } \\
\text { interval }\end{array}$ \\
\hline $\begin{array}{l}\text { Syphilis } \\
\text { Neisseria gonorrhoea } \\
\text { Trichomonas vaginalis } \\
\text { Candida albicans } \\
\text { At least one of the above genital infections, } \\
\text { candidiasis excepted }\end{array}$ & $\begin{array}{l}1.62 \\
1.24 \\
4.98 \\
6.97 \\
6.59\end{array}$ & $\begin{array}{l}1.58 \\
0.34 \\
1.92 \\
4.59 \\
3.50\end{array}$ & $\begin{array}{l}1 \cdot 0 \\
3 \cdot 7 \\
2 \cdot 6 \\
1 \cdot 6 \\
1 \cdot 9\end{array}$ & $\begin{array}{l}0 \cdot 5-2 \cdot 2 \\
1 \cdot 0-13 \cdot 3 \\
1 \cdot 5-4 \cdot 6 \\
1 \cdot 1-2 \cdot 4\end{array}$ \\
\hline
\end{tabular}

^Incidences were computed per 100 women-months, excluding women with syphilis at inclusion. 


\section{Discussion}

In Kigali, women attending prenatal clinics are considered to be representative of women of reproductive age: $92 \%$ of urban pregnant women seek prenatal medical services during the last trimester of pregnancy, ${ }^{12}$ only $2 \%$ of Rwandan women are sterile, ${ }^{13}$ less than $14 \%$ of Rwandan women use a modern contraceptive method ${ }^{12}$ and the fertility rate is one of the highest in the world ( 8.6 children per woman at the end of her childbearing years). ${ }^{12}$ In addition, only $8 \%$ of the eligible women refused to participate to this study and the $\mathrm{CHK}$ is the only hospital of the city. Overall, our sample is considered to be representative of adult urban Rwandan women, allowing us to estimate the prevalence of HIV and genital infections in this population.

The HIV seroprevalence observed was the highest figure ever reported since the beginning of the HIV epidemic in Rwanda and in Africa. Bearing in mind the seroprevalence rate of $21 \%$ in 1986 among urban women, ${ }^{2}$ the HIV seroprevalence rate of $34 \%$ in 1993 among urban pregnant women suggests the continuing progression of the HIV epidemic in this population. Furthermore, the Rwandese serosurveillance system has also shown that HIV seroprevalence among urban adults has increased in recent years in Kigali. ${ }^{14}$ Finally, a seroincidence study in HIVwomen of reproductive age has found in the same population an annual incidence around 3 to $5 \% . .^{15}$

Regardless of HIV infection, the prevalence at inclusion of genital infections was high in pregnant women. Genital infections are clearly a public health problem in this population. The prevalence of syphilis is high as it is in numerous countries of Africa, such as Kenya, ${ }^{16}$ Mozambique, ${ }^{17}$ Cameroon ${ }^{18}$ and Uganda. ${ }^{19}$ This figure was $4.4 \%$ in the same population in $1980 . .^{20}$ The prevalence of gonorrhoea seemed to be lower than the rate of $13 \%$ estimated in 1988 among HIV+ women from the same population. ${ }^{21}$ These high prevalence figures confirm, first, the weakness of prevention programmes against genital infections and may explain the explosive AIDS epidemic in many developing countries including Rwanda. ${ }^{22} 23$ Second, we know that adverse neonatal and maternal complications may be associated with maternal syphilis, gonorrhoea or Chlamydia trachomatis infections during pregnancy. Chlamydia trachomatis seemed to be less important than anticipated in our population. In front of this heavy public health burden, our findings suggest that prenatal care should include in Kigali at least once a systematic screening for syphilis and clinical genital examination with speculum and in the presence of signs or symptoms, a laboratory work-up looking for syphilis, gonorrhoea, trichomoniasis or candidiasis.

We consider that our estimation of incidence of genital infections was a minimum estimate because laboratory screening was not systematically repeated for cost reasons. However, the two groups of HIV+ and HIV women being similar in terms of age, parity, marital status and follow-up procedures, our comparison group of HIV - women allowed us to study prospectively the association between genital infections and HIV infection and more specifically the impact of HIV infection on the occurrence of genital infections.

$\mathrm{HIV}+$ women had more genital infections at inclusion (Neisseria gonorrhoea and Trichomonas vaginalis) than HIV - women. However, this cross-sectional analysis does not allow us to establish a causal relationship between STDs and occurrence of HIV infection. Several studies have already proven that in Africa, STDs and particularly genital ulcers have the most consistent and strongest association with HIV infection. ${ }^{24-28}$ Heterosexual transmission accounts for most HIV infections in Africa and other STDs play the role of co-factors in spreading HIV infection with the same risk behaviour. ${ }^{41625262930}$

The longitudinal part of our study, controlling the temporal sequence of events, showed that HIV infection was associated with an increased occurrence of certain genital infections. We did not find any impact of HIV infection on syphilis incidence, contrary to several epidemiological studies. ${ }^{25}$ This may be explained by a decrease of the reliability of the RPR reaction with the degree of immunodeficiency. ${ }^{1031}$ or the lack of power because of the low number of events in our study. HIV infection tended to be associated with an increased occurrence of gonorrhoea. This is in agreement with other observations in Africa where the incidence of gonorrhoea ranged between $1 \%$ and $23 \% .^{21} 2532 \mathrm{HIV}$ infection had a strong impact on the incidence of Trichomonas vaginalis infection in our study, even if the figures reported here are lower than those obtained in Kinshasa, Zaire. ${ }^{32}$ In addition, we found that HIV infection seemed to facilitate relapse of trichomoniasis after treatment. This could be due to reinfection or a failure of treatment. For Chlamydia trachomatis, our observations were discordant with those reported in other populations in which the prevalence and also incidence were associated with HIV infection. ${ }^{171929}$ The reason for this difference remains unclear but diagnostic techniques vary from one study to the other.

When controlling for the HIV serostatus, a profound immunodeficiency was not a risk factor for developing genital infection. However, our findings suggest several hypotheses for increased incidence of genital infections among HIV+ women in comparison to HIV - women: more frequent at risk sexual practices, poor compliance to treatment or increased susceptibility to infectious agents.

We documented clearly in our sample high HIV seroprevalence and high prevalence of other genital infections. These results suggest the limited effectiveness of prevention programmes against AIDS and STDs in this population and the usefulness of serosurveillance among pregnant women. Controlling the spread of HIV infection in women is of utmost importance because a large number of HIV+ women of reproductive age will result 
in a higher number of children with perinatally acquired HIV infection. ${ }^{3}$ Integration of STDs and HIV control programmes into maternal and child health care and family planning services should be useful in the aim of prevention and control of STDs and HIV transmission in Africa. ${ }^{33}$ Furthermore, pregnancy may be a unique opportunity for the treatment of STDs among women. STDs management may be integrated into prenatal care in Africa, using simple algorithms based on a syndromic case management without the systematic need of laboratory tests when they are not available on a large scale. ${ }^{33}{ }^{34}$ However, syphilis screening should be systematically offered to every pregnant woman as already demonstrated elsewhere. ${ }^{16}$ This study underlines once more the necessity for quick, inexpensive and reliable diagnostic tests for STDs. Further studies are in progress in this cohort, to measure the impact of HIV infection on pregnancy outcome, after treatment of other STDs.

The authors would like to dedicate this paper to the women participating to the study, the EGE staff and the many people involved in this project in Kigali and who have paid now a heavy tribute to the 1994 civil war in Rwanda. We give special thanks to the Rwandan National AIDS Program for their support to the study as well as the following persons: Drs Antoine Serufilira (WHO, Kigali), Manbu Ma Disu (WHO Kigali/Geneva) and Benjamin Nkowane (WHO Geneva).

The EGE Study Group comprises the following: Gynaecology and Obstetrics: A De Clercq (principal investigator), A Cyiamana, M Mudaheranwa $t$, $C$ Munyangabe, J Ntawuruhunga $t$, B Ntezayabo, $M$ Nyiraziraje, C Zilimwabagabo. Microbiology: J Bogaerts, E Karita, A Simonon, P Van de Perre. Epidemiology: F Dabis, C Gazille, J Ladner, V Leroy, P Msellati, R Salamon.

t Deceased.

This study was presented in part at the VIIIth International Conference on AIDS in Africa, Marrakech, Marocco, 12-16 December 1993 (abstract No THRT 033 239). This study was funded by the World Health Organization Global wrogramme on AIDS, the Agence Nationale de Recherches sur le SIDA (France), the Belgian and French Medical Cooperations.

1 World Health Organisation, Global Programme on AIDS The HIVIAIDS pandemic: 1993 Overview. Geneva: WHO/GPA/CNP/EVA/93.1, 1993:17p.

2 Rwandan HIV seroprevalence study group. Nationwide community-based serological survey of HIV-1 and other human retrovirus infections in a central African country. Lancet 1989;i:941-4.

3 Chin J. Current and future dimensions of the HIV/AIDS pandemic in women and children. Lancet 1990;336: $221-4$.

4 Mertens T, Hayes R, Smith P. Epidemiological methods to study the interaction between HIV infection and other sexually transmitted diseases. AIDS 1990;4:57-65.

5 St Louis M, Kamenga M, Brown C, et al. Risk for perinata HIV-1 transmission according to the maternal immunologic, virologic and placenta factors. $¥ A M A$ 1993;269: 2853-9.

6 Dabis F, Ladner J, De Clercq A, et al. Pregnancy outcome in HIV infected women. A cohort study in Kigali (Rwanda), 1992. IXth International Conference on AIDS. Berlin, Germany, 6-11 June 1993: abstract WSBO61.

7 Ladner J, De Clercq A, Nyiraziraje M, et al. HIV seroprevalence and counselling in pregnant women. A cohort study in Kigali (Rwanda), 1992. IXth cohort study in Kigali (Rwanda), 1992. IXth International Conference on AIDS. B

8 Centers for Disease Control. Interpretative criteria used to report Western Blot results for HIV-1 antibody testingUnited States. MMWR 1991;40:692-5.

9 World Health Organisation. Provisional clinical case definition for AIDS. Wkly Epidemiol Rec 1986;61:224.

10 Centers for Disease Control. Recommendations for diag nosis and treating syphilis in HIV infected patients. MMWR 1988:37:600-8.

11 Ursi J, Van Dyck E, Van Houtte C, et al. Syphilis in Swaziland. A serological survey. Br f Venereal Dis 1981; 57:95-9.

12 République du Rwanda. Office National de la Population Enquête Démographique et de Santé au Rwanda. Kigali: Office National de la Population, 1992:22p.

13 République du Rwanda. Office National de la Population. Recensement Général de la Population et de l'Habitat, 15 août 1991: analyse des résultats Kigali, République du Rwanda, 1994:226.

14 Bucyendore A, Van de Perre P, Karita E, Nziyumvira A Sow I, Fox E. Estimating the seroincidence of HIV-1 in the general adult population in Kigali, Rwanda. AIDS the general adult

15 Leroy V, Van de Perre $P$, Lepage $P$, et al. Seroincidence of HIV-1 infection in African women after a delivery: a prospective cohort study in Kigali, Rwanda; 1988-1992. AIDS 1994;8:983-6.

16 Temmerman $M$, Ali F, Ndinya-Achola J, Moses S, Plummer F, Piot P. Rapid increase of both HIV-1 infection and syphilis among pregnant women in Nairobi,
Kenya. AIDS 1992;6:1181-5.

17 Vuylsteke B, Bastos R, Barreto J, et al. High prevalence of sexually transmitted diseases in a rural area in Mozambique. Genitourin Med 1994;69:427-30.

18 Zekeng L Yanga D, Trebucq A. HIV prevalence in patients with STDs in Yaounde in 1989 and 1990 necessity of STD control programme. Genitourin Med 1992;68:117-9.

19 Wagner H, Van Dyck E, Roggen E, et al. Seroprevalence and incidence of sexually transmitted diseases in a rura Ugandan population. Int $\mathcal{F}$ STD AIDS 1994;5:332-7.

20 De Clercq A. Problèmes en Obstétrique et Gynécologie. In: Meheus A, Butera S, Eylenbosch W, Gatera G, Kivits M, Musafili I, eds. Santé et Maladie au Rwanda. Administration Générale de la Coopération au Développement. Bruxelles: 1982:651.

21 Allen S, Serufilira A, Bogaerts J, et al. Confidential testing and condom use promotion in Africa; impact on HIV and condom use promotion in Africa; impact on

22 Piot $P$, Laga $M$, Ryder $R$, et al. The global epidemiology of HIV infection: continuity, heterogeneity, and change. f Acquir Immune Defic Syndr 1990;3:403-12.

23 De Schryver A, Meheus A. Epidemiology of sexually transmitted diseases: the global picture. Bull WHO 1990 68:639-54.

24 Cameron D, Simonsen J, D'Costa L, et al. Female to male transmission of Human Immunodeficiency Virus type 1: risk factors for seroconversion in men. Lancet 1989 , ii:403-7.

25 Laga M, Nzila N, Goeman J. The interrelationship of sexually transmitted diseases and HIV infection: implications for the control of both epidemics in Africa. AIDS 1991;5(suppl 1):S55-63.

26 Laga M, Diallo M, Buvé A. Inter-relationship of sexually transmitted diseases and HIV: where are we now? AIDS 1994;8(suppl 1):S119-24

27 Latif A. Sexually transmitted diseases in Africa. Genitourin Med 1990;66:235-7.

28 Plourde P, Plummer F, Pepin J, et al. Human Immunodeficiency Virus infection in women attending a sexually transmitted diseases clinic in Kenya. $\mathcal{F}$ Infect Dis 1992; 166:86-92.

29 Laga $M$, Manoka A, Kivuvu $M$, et al. Non-ulcerative sexually transmitted diseases as risk factors for HIV-1 transmission in women: results from a cohort study. transmission in women:

30 Piot P, Kreiss J, Ndinya-Achola J, Ngugi E, et al. Heterosexual transmission of HIV. AIDS 1987;1 199-206.

31 Johnson P, Graves S, Stewart L, Warren R, Dwyer B, Lucas R. Specific syphilis serological tests may become negative in HIV infection. AIDS 1991;5:419-23.

32 Nzila N, Laga $M$, Thiam $M$, et al. HIV and other sexually transmitted disease among female prostitutes in Kinshasa. AIDS 1991;5:715-21.

33 Merson M. Slowing the spread of HIV: agenda for the 1990s. Science 1993;260:1266-8.

$34 \mathrm{La}$ Ruche G, Lorougnon F, Digbeu N. Therapeutic algorithms for the management of sexually transmitted diseases at the peripheral level in Côte d'Ivoire, West Africa: assessment of efficacy and cost. Bull WHO 1995 (in press). 